\title{
Retinal toxicity to antimalarial drugs: chloroquine and hydroxychloroquine: a neurophysiologic study
}

This article was published in the following Dove Press journal:

Clinical Ophthalmology

7 March 2012

Number of times this article has been viewed

\author{
Donald F Farrell \\ University of Washington School \\ of Medicine, Seattle, WA, USA
}

Correspondence: Donald Farrell Professor Emeritus, Neurology, Former Director, EEG and Clinical Neurophysiology, University of Washington School of Medicine, Seattle, WA 98195, USA

Tel +l 2064374026

Email donf@u.washington.edu

\begin{abstract}
Over a 30-year period, 29 cases of antimalarial retinal toxicity were studied in a tertiary medical center. Three cases of chloroquine and 26 cases of hydroxychloroquine toxicity were studied. A number of these cases were studied before multifocal electroretinogram (mfERG) became available and show how insensitive the corneal full-field flash ERG is in diagnosing this condition. It became apparent that even mfERG failed to diagnose some early patients who either had an abnormal fundus examination or Humphrey's automated perimetry (protocol 10-2). The age of the patient and the number of years of exposure to antimalarial drugs appears to be directly related to the development of this retinal disorder. All three of the "quantitative retinal tests" recommended in the "Guidelines" - mfERG, spectral domain optical coherence tomography (SD-OCT), and autofluorescence - fail to identify all of the cases of antimalarial retinal toxicity. mfERG is probably the most sensitive of the three tests, but no direct comparison has yet been accomplished. None of these "quantitative tests" appear to provide the "gold standard" necessary for detecting early hydroxychloroquine retinal toxicity.
\end{abstract}

Keywords: chloroquine, hydroxychloroquine, multifocal electroretinogram (mfERG), spectral domain optical coherence tomography (SD-OCT), autofluorescence

\section{Introduction}

In 2011, the American Academy of Ophthalmology provided revised recommendations for the testing of patients taking antimalarial drugs. ${ }^{1}$ The goal of these guidelines is to identify antimalarial retinal toxicity in its earliest stages so that the offending drugs can be stopped as early as possible, when clinically indicated. Recommended screening procedures include: "a baseline dilated fundiscopic examination (with and with/out fundiscopic photography) and a Humphrey's automated perimetry (protocol 10-2). After 5 years of treatment these tests should be accomplished yearly". When these screening tests become abnormal, consultation with the referring rheumatologist concerning the possibility of stopping the medication should be carried out. Quantitative studies should then be carried out. These include multifocal electroretinogram (mfERG), spectral domain optical coherence tomography (SD-OCT), and fundus autofluorescence. When available, mfERG appears to be the most sensitive test followed by autofluorescence. Clinicians should be aware that each test has a certain percentage of false negatives. In the case of a normal examination and a high suspicion for antimalarial toxicity, a second quantitative test should be accomplished.

The purpose of this manuscript is to show that each objective test as carried out at this time may show false negative results and that a dilated fundiscopic examination, Humphrey's automated perimetry, and clinical judgement should decide when to stop 
using the offending agent. Objective tests should be used for confirmation of patients with abnormal screening tests. In future guidelines, an additional criteria should be added: that an individual to receive yearly screenings should be over the age of 40 years and have at least 5 years of exposure to standard doses of hydroxychloroquine.

\section{Methods}

This study represents the experience of a tertiary care center providing visual neurophysiologic testing of patients from four states: Washington, Alaska, Montana, and Idaho. Patients suffering from chloroquine and hydroxychloroquine retinal toxicity were referred for study. All of the studies were carried out between 1981 and 2011, giving a 30-year experience. This is a retrospective study of these patients. The patient population included three cases of chloroquine and 26 cases of hydroxychloroquine retinal toxicity. All patients were female, 26 of the cases were over 40 years of age and suffered from a variety of collagen vascular disorders, including: four cases of SLE - systemic lupus erythematosus; ten cases of rheumatoid arthritis; four cases of Sjögren's syndrome; one case of chronic discoid lupus; one case of dermatomyositis; and nine cases of undifferentiated collagen vascular disease. Mean age at the time of study: chloroquine retinopathy, 64.7 years (range 40-67 years); hydroxychloroquine retinopathy, 63.7 years (range 38-68 years). The duration of treatment: patients on chloroquine, 13.3 years (range 10-16 years); patients on hydroxychloroquine, 8.0 years (range 3-20 years). Twenty-four patients complained of decreased vision, two had no visual complaints, and three complained of blurred vision. All patients received the following doses of the antimalarial medication (approximately $80 \%$ of this population received $400 \mathrm{mg}$ /day hydroyxchloroquine in divided doses, the remaining $20 \%$ were either on lower doses of $200 \mathrm{mg} /$ day, alternate doses $200 \mathrm{mg} /$ day with $400 \mathrm{mg} /$ day, or finally one individual who was on a higher dose at $600 \mathrm{mg} /$ day). No patient weights were recorded so the dose $/ \mathrm{kg}$ could not be calculated.

Until mfERG became clinically available, patients were studied with corneal full-field flash ERGs. Two of the three patients with chloroquine retinopathy were studied with corneal full-field flash ERG, the third case with mfERG. Eighteen patients with hydroxychloroquine toxicity were examined by mfERG. Serial studies were accomplished in two patients. One of the patients with chloroquine and one with hydroxychloroquine were also studied with electrooculograms, only the patient with chloroquine was abnormal, the other had a perfectly normal response. Decreased vision was the most common complaint. Fundiscopic retinal changes were seen in 20 cases, including one case of drusen. Seven cases were reported to have no retinal changes. Visual field examination was reported in ten cases and corneal deposition of hydroxychloroquine was described in two patients.

Corneal full-field flash ERGs, and electro-oculograms, were accomplished by methods previously reported. ${ }^{2}$ The mfERGs were accomplished using Sutter Electronic Data Interchange (EDI) equipment (Sutter Health, Sacramento, CA) and Veris Corporation software. The software provides three levels of analysis, 103 kernals, ring summation, and a three-dimensional plot of the data. Luminescence was set at $197 \mathrm{~cd} / \mathrm{m}^{2}$ for white hexagons and $1 \mathrm{~cd} / \mathrm{m}^{2}$ for the black hexagons. First-order kernals were utilized for interpretation.

\section{Results}

Dilated fundiscopic examination showed abnormalities in all the cases where this information was available. The most common retinal change included a Bull's eye or Target maculopathy in both chloroquine and hydroxychloroquine retinal toxicity. Fundiscopic photos of the changes seen in chloroquine and hydroxychloroquine retinopathy are shown in Figure $1 \mathrm{~A}$ and $\mathrm{C}$.

The earliest of the current studies used the corneal fullfield flash ERG as the quantitative diagnostic test, as the multifocal ERG and other quantitative tests had not yet been developed. In all, there were nine individuals who underwent testing with corneal full-field ERG. There were two

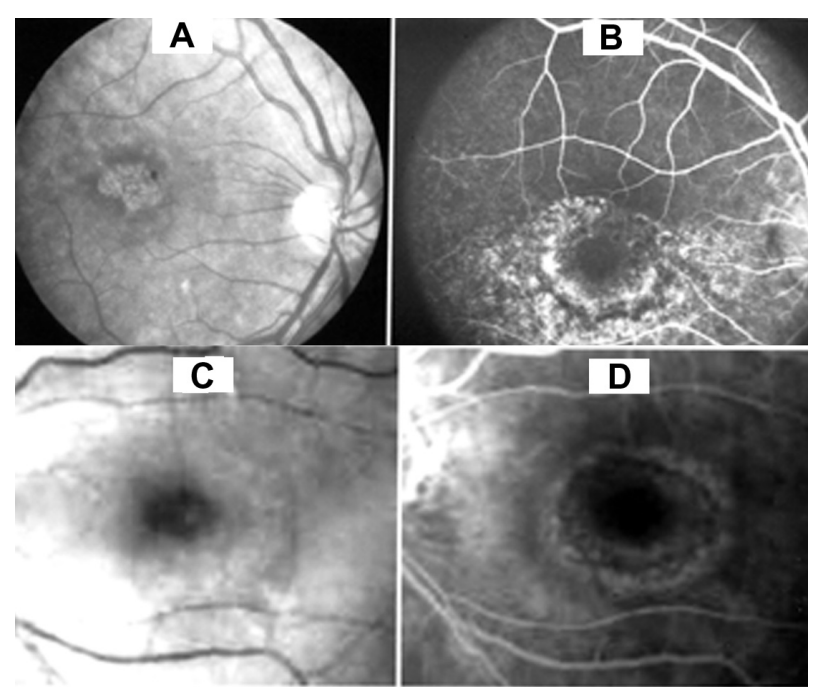

Figure I (A) Fundiscopic photo of the retina showing a target maculopathy in a patient with chloroquine retinopathy. (B) Fluorescein angiography of same case showing a fairly large area of dye leakage extending from the perimacular region. (C) Fundiscopic photo of the target maculopathy in a patient with hydroxychloroquine retinal toxicity. (D) Fluorescein angiography of this case shows a milder change than seen with chloroquine. 
individuals with chloroquine exposure that showed markedly abnormal ERGs, one with both rods and cones being about equally affected and the second with cones being affected greater than rods. The first case showed a near complete loss of all retinal function (end stage retinopathy). Both of these individuals had abnormal visual field examinations.

Seven individuals with hydroxychloroquine retinal toxicity underwent corneal full-field flash ERGs with three being abnormal and four being normal. The three abnormal individuals were rated from mildly to moderately abnormal. The abnormalities consisted primarily of decreased B-wave amplitude with normal implicit times. Cones and rods were both affected. One of the individuals with an abnormal corneal full-field flash ERG also had an abnormal visual field examination. Two of the four normal individuals with corneal full-field flash ERGs had abnormal visual field examinations. All of these nine individuals had abnormal fundiscopic examinations with macular pigmentary change, with Bull's eye and Target maculopathy being the most common findings described. With the advent of mfERG, the percentage of abnormal cases increased from about $42 \%$ using corneal full-field ERGs to approximately $72 \%$ using mfERGs. In summary, corneal full-field flash ERGs are limited in ability to identify early macular changes and require that the disorder is so severe that widespread retinal dysfunction is invariably present.

Eighteen individuals, one was exposed to chloroquine and seventeen to hydroxychloroquine, underwent $\mathrm{mfERG}$ testing which is much more specific for examining the central $40^{\circ}$ of the retina, $20^{\circ}$ on each side of the fixation point. One of the potential problems with mfERGs is that Verius Corporation, which produces the hardware and software, provides built-in normative data. The normative data does not appear to take into account changes in the test that occurs with advancing age. In our laboratory, a very small sample of asymptomatic "normal" adults at 20, 40, and 60 years of age were studied, and the mfERG demonstrated a definite change in amplitude and implicit time with age. Between 20 and 40 years there is an amplitude drop of about $50 \%$, between 40 and 60 years the response slows somewhat, but has an amplitude less than at 40 years of age (Figure 2). From 20 to 60 years of age the implicit time increases from 25 to 29 milliseconds. Since the vast majority of patients suffering from antimalarial toxicity are older, age-related normative data should be generated for future diagnostic use. In order to maximize the value of mfERG, future normative studies of at least ten individuals per decade from 20 to at least 70 years should be carried out.
One patient who had been exposed to chloroquine showed severe widespread mfERG changes (Figure 3B). Seventeen individuals with hydroxychloroquine toxicity were studied with mfERGs. Twelve cases showed the typical loss of central macular dysfunction (Figure 3C). Five cases were found to be normal. Of these five cases, two had reduced amplitudes thought to be related to age. In those cases that had visual field testing and fundiscopic examination, 100\% were abnormal. mfERG was abnormal in $72 \%$ of the hydroxychloroquine exposed individuals. Serial studies were carried out in two patients with hydroxychloroquine toxicity. In the first case, a series of studies was carried out over a 3-year period. No worsening of the mfERG or clinical condition over this period was documented. The second case underwent serial studies over a 10-month period and progression was documented (Figure 4) along with worsening of the visual field examination (Humphrey's 10-2 protocol) in spite of having stopped the offending drug. In two cases, found normal by mfERG, the fundiscopic examination showed macular pigmentary changes. In one of the two cases found normal by mfERG, the perimetric examination was also abnormal. One additional patient who was reported to have a normal mfERG had a normal fundiscopic examination and an abnormal perimetric examination, so that two mfERG normal individuals had evidence of the retinal disorder.

\section{Discussion}

The incidence and prevalence of antimalarial retinal toxicity is unknown. No population studies have ever been reported. In our tertiary diagnostic laboratory, during the same time period that 29 individuals with antimalarial toxicity were referred, there were 267 cases of retinitis pigmentosa. This means that retinitis pigmentosa is approximately ten times more common than antimalarial retinal toxicity. The incidence of retinitis pigmentosa is reported to be $0.25: 1,000$ individuals. One can calculate a relative prevalance for antimalarial retinal toxicity at about $0.025: 100,000 . .^{3-5}$ Based on the variable incidence and prevalence data for the collagen vascular disorders in the literature, it is difficult to calculate the exact risk to patients with rheumatoid arthritis and SLE treated with antimalarials, but from our data it appears that less than $1 \%$ of the patients treated with antimalarials are at risk to develop retinal toxicity. The National Arthritis Data Workgroup provide approximate prevalence data for rheumatoid arthritis in women at $1.06 \%$ of the population and SLE to be from $0.05 \%$ to $0.1 \%$ of the population. The percentage of women with rheumatoid arthritis at risk for hydroxychloroquine retinal toxicity is about $0.8 \%$ while for 

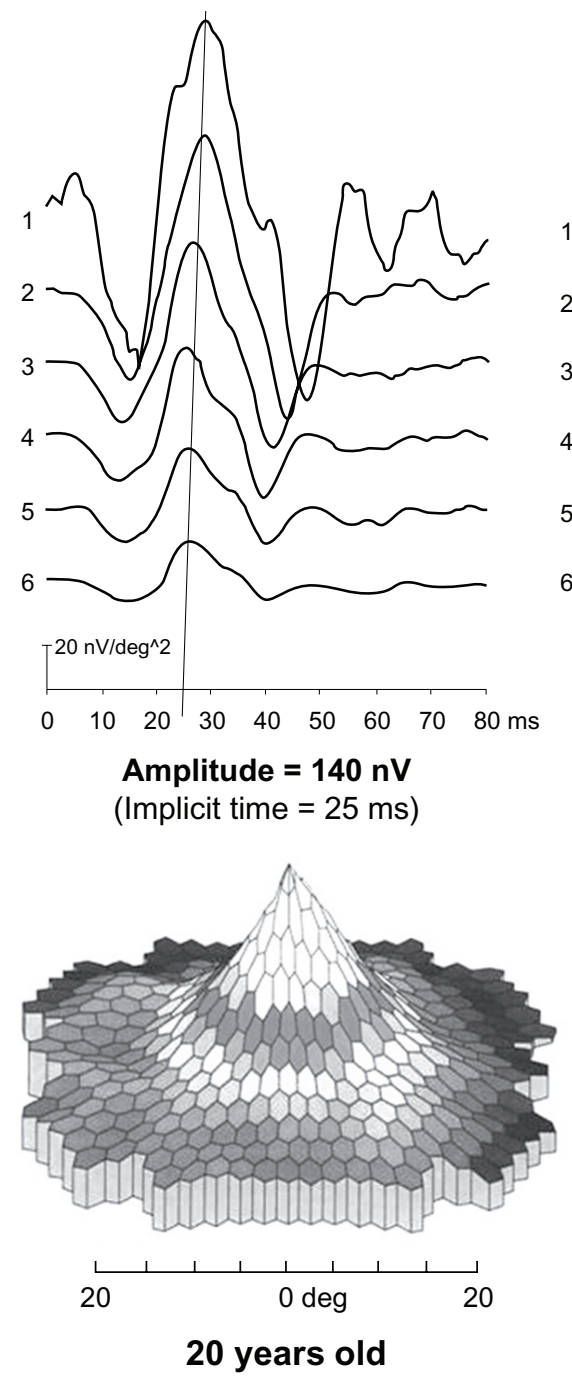

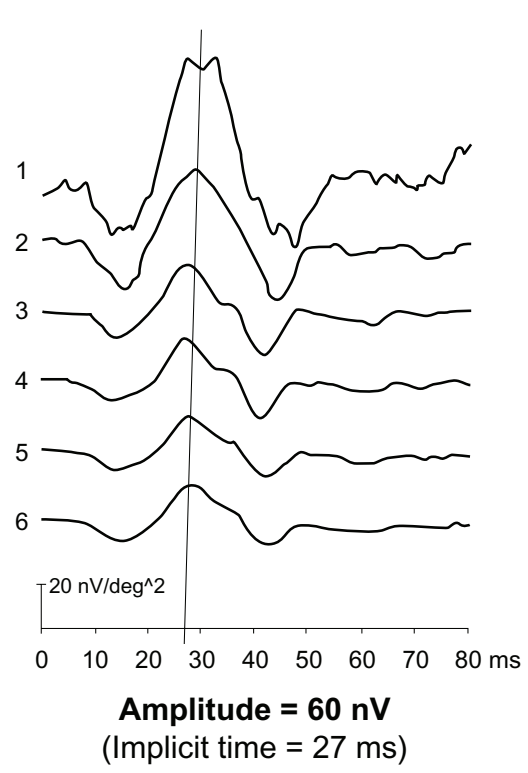

Implicit time $=27 \mathrm{~ms}$

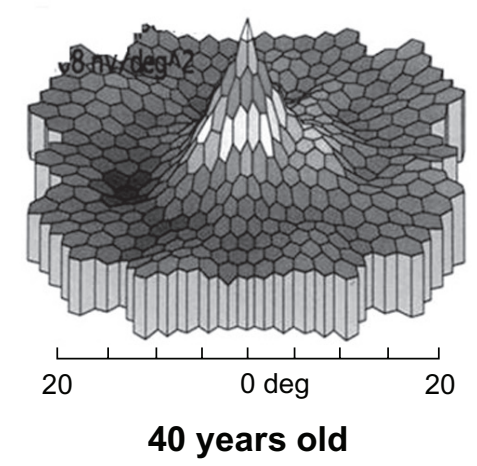

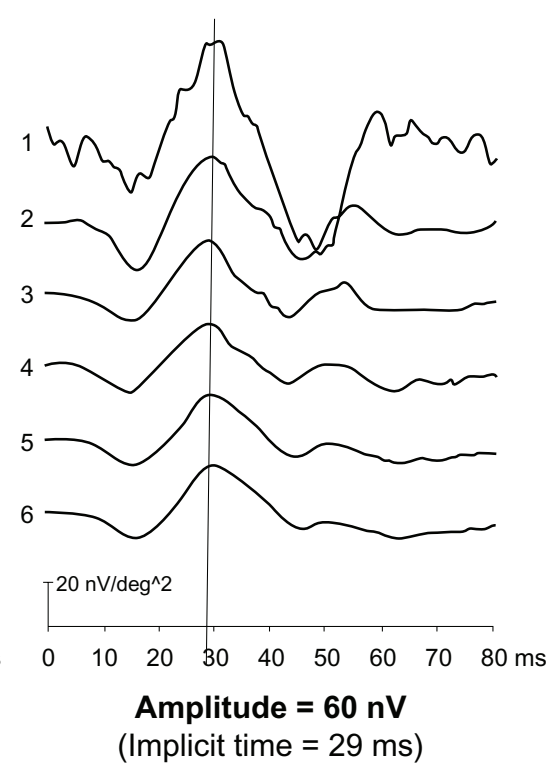

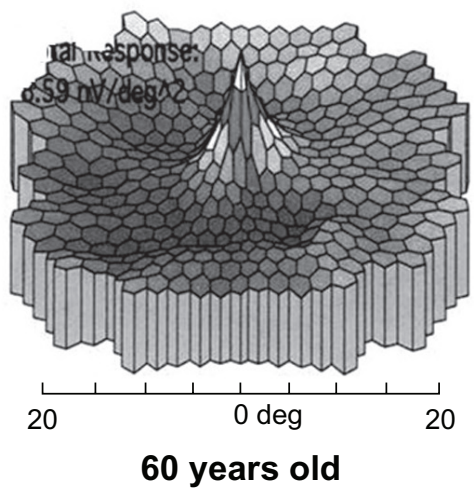

Figure 2 Age-related changes seen in mfERG testing. The upper part shows the quantitative ring analysis while the lower part shows the three-dimensional analysis. Approximately $50 \%$ of the response is lost between 20 and 40 years of age. The response stabilizes between 40 and 60 years of age and little further loss occurs. The implicit time increases from 25 to 29 milliseconds over the 40 years.

those women with SLE, the percentage is lower and ranges from $0.04 \%$ to $0.08 \%$. Our current data on the frequency of hydroxychloroquine retinal toxicity agrees well with that reported by Tzekow. ${ }^{6}$

The vast majority of patients with rheumatoid arthritis develop symptoms after 40 years of age or older, ${ }^{7,8}$ while patients with SLE generally are symptomatic earlier in life, the greatest number being during their reproductive age. ${ }^{9,10}$ The age of onset for Sjögren's disease is generally 45-55 years of age. ${ }^{11}$ The differences in the percentage of patients developing antimalarial retinal toxicity most likely relate to the different ages of onset; rheumatoid arthritis and Sjögren's disease patients generally being older. The mean age for the patients with SLE was 50.3 years and each had many years of exposure ( $>15$ years) to hydroxychloroquine $400 \mathrm{mg} /$ day in divided doses. The mean age for patients with rheumatoid arthritis was 56.4 years and each had a shorter length of exposure to antimalarial drugs than the SLE cases, two of the five individuals having been exposed from 4 and 8 years respectively. The mean age for Sjögren's disease was intermediate between the other two disorders, at 54 years.

Since the purpose of documenting that an individual has antimalarial retinopathy, diagnostic tests must identify affected individuals as early as possible in order to be able to stop these medications before significant retinal changes have occurred. It is clear that the corneal full-field flash ERG has no place for the testing of chloroquine or hydroxychloroquine retinal toxicity. It is not sensitive enough to pick up early focal maculopathies. In fact, almost all focal maculopathies have an either normal or near normal corneal full-field flash ERG. This procedure remains an excellent choice for the diagnosis of the diffuse retinopathies, such as retinitis pigmentosa. 


\section{A}

Normal

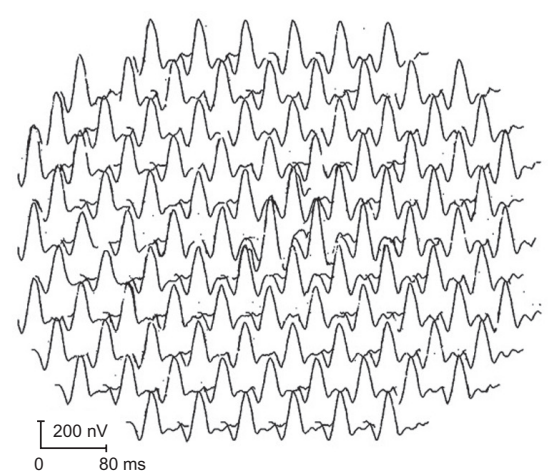

B

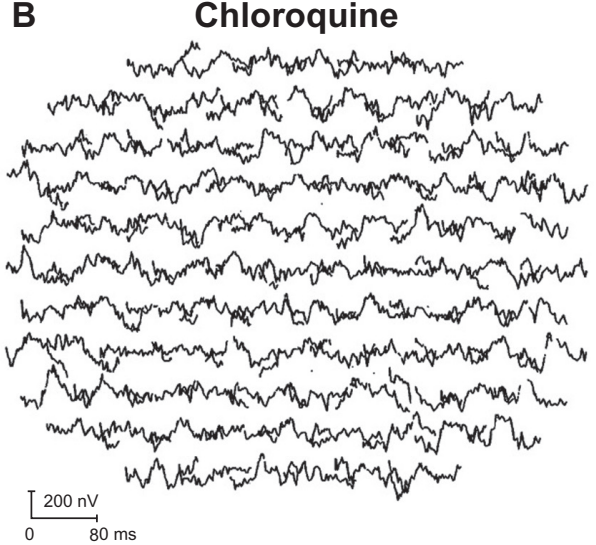

C Hydroxychloroquine

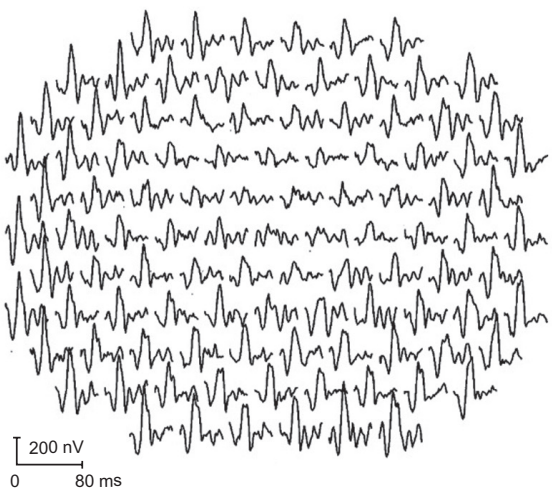

Figure 3 (A) Normal responses of the 103 kernals provided by the Veris Corp (The Veris Corporation, McLean, VA) as part of the mfERG package. (B) Widespread loss to all responses in the $40^{\circ}$ field of testing in a patient with relatively advanced chloroquine retinal toxicity. (C) Focal reduction in the central macular region with normal responses at the periphery in a patient with typical hydroxychloroquine retinal toxicity.

The individuals suffering from chloroquine exposure have much more severe retinal changes than seen in the typical cases of hydroxychloroquine toxicity. Chloroquine tends not only to affect the macular region of the retina, but causes widespread dysfunction of the entire retina. Its clinical use has stopped for the most part and hydroxychloroquine has replaced it for the treatment of collagen vascular disorders and as an antimalarial agent in Africa and other parts of the world.

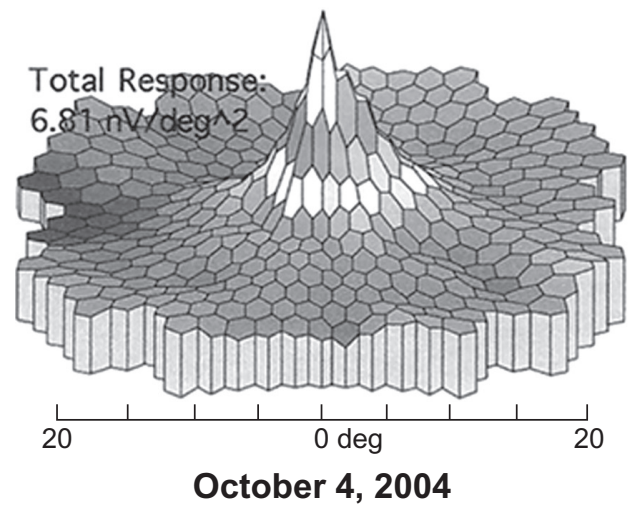

Multifocal ERGs are much more sensitive in diagnosing antimalarial retinal toxicity than corneal full-field flash ERGs. ${ }^{12-15}$ This test specifically examines the macular and perimacular regions of the retina (central $40^{\circ}$ ). However, in this study mfERG failed to identify at least four individuals with hydroxychloroquine toxicity. This means that approximately $28 \%$ of individuals with hydroxychloroquine retinal toxicity would be false negatives. That is a high percentage

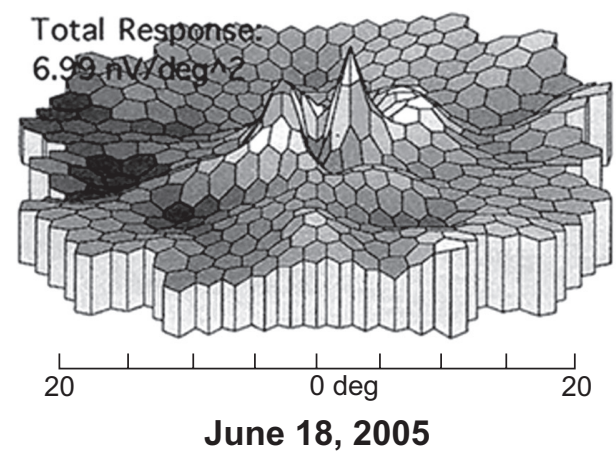

Figure 4 Results of a serial study taken over 10 months after patient stopped taking hydroxychloroquine. The panel on the right shows a dramatic reduction in the central responses that occurred despite stopping the medication. 
for any type of diagnostic test. This finding raises the question of how sensitive mfERG test is in diagnosing very early antimalarial retinal toxicity. In this current study perimetry, whether it be Goldman's or Humphrey's automated perimetry, identified nearly $100 \%$ of the cases and should remain the "gold standard" until more data have been collected for $\mathrm{mfERG}$ and the other two new diagnostic techniques, SD-OCT ${ }^{15}$ and fundus autofluorescence. ${ }^{16}$

In one study, ${ }^{15} 24$ women with significant hydroxychloroquine exposure were studied. Only six of the 24 had visual complaints of decreased visual acuity. All 24 underwent fundiscopic examination, Humphrey's automated perimetry and spectral domain optical tomography. Fundiscopic examination revealed eleven pigmentary abnormalities, one with drusen, and 12 with normal fundiscopic examinations. HVF-10-2 protocol found 13 cases to be abnormal, and eleven normal cases. The most common abnormality included scotomata of various severities. Five abnormalities were identified with SD-OCT. In three of the abnormal cases, a Target maculopathy was identified, one case had retinal pigment epithelial changes on fundiscopic examination, but had a scotoma on perimetric examination. A single case with a reported normal fundiscopic examination had a paracentral scotoma, OU. At least two false negatives were reported. One case had clinical complaints, central scomata OS $>$ OD by perimetry, a normal fundus examination and a negative SD-OCT examination. The second case had a complaint of decreased visual acuity, a paracentral scotoma, OS $>$ OD by perimetry, mild macular puckering on fundiscopic examination and a negative SD-OCT. In this study, four individuals showed scotomata by perimetry in which the SD-OCT was normal. False negatives appear to be even higher for SD-OCT than for mfERG testing.

Fundus autofluorescence was compared with mfERG in the early detection of retinopathy in chloroquine and hydroxychloroquine retinal toxicity. ${ }^{16}$ In this study there were ten individuals who had a normal Fundus Autofluorescence (FAF) and mfERG, five individuals who had a normal FAF and abnormal mfERG, two individuals who had an abnormal FAF and normal mfERG, and eight individuals where both the FAF and mfERG were abnormal. Fundus autofluorescence changes included "pericentral ring of increased FAF" and "mottled pericentral loss of FAF with adjacent increased in FAF". mfERG abnormalities included decreased amplitude of the central kernals with peripheral responses being normal. It is clear that FAF studies do not identify all of the cases of early antimalarial retinal toxicity, but may pick up a small number of individuals not identified by mfERG.
None of the three major tests as currently accomplished, mfERG, SD-OCT, and fundus autofluorescence provide the sensitivity necessary to meet the objective criteria for the recently proposed guidelines ${ }^{1}$ of early identification of individuals suffering from antimalarial retinal toxicity. The non-objective tests, Humphrey's 10-2 protocol automated perimetry and fundiscopic examination and photography continue to provide the highest yields of early cases and drug stoppage when indicated should be based on these studies. Objective testing should then be used to confirm the presence of antimalarial retinal toxicity knowing that a few affected individuals will show normal results. mfERG appears to be the most sensitive of the three objective tests, but in the face of a normal test when there is a high suspicion from the dilated fundiscopic examination and perimetry, a second test should be performed and fundus autofluorescence is probably the best choice in this situation. Serial studies may also be helpful in these cases as progression of retinopathy may occur in some of the affected patients after the offending medication has been stopped.

At some point, a head-to-head comparison of the three quantitative tests should be carried out.

The pathophysiology of antimalarial retinal toxicity includes dysfunction of the lysosomes at the retina-retinal pigment epithelium (RPE) interface. ${ }^{17}$ However, that would not explain the age population in which this condition develops. Virtually all cases are older than 40 years and have had exposure of greater than 5 years. In this mfERG study, there is a dramatic drop in retinal neurons between the ages of 20 and 40 years of age. It is likely that this loss of nerve cell reserve sets the stage for the clinical development of antimalarial retinal toxicity. The failure for toxic retinopathy to occur in younger individuals is most likely related to the fact that those individuals have a significant neuronal reserve which prevents the development of clinical disease for many years.

Currently, mfERG, SD-OCT and autofluorescence studies should be utilized to objectively diagnose antimalarial retinal toxicity, knowing that a certain percentage of early cases will be missed, even when more than one test is utilized. Serial studies remain a valuable tool in following the progression of the disease. One additional factor which may predispose to this condition is the observation by $\mathrm{mfERG}$ that there is a large loss of responding neurons between 20 and 40 years of age. It is this loss of neurons that may predispose the older individuals to develop this disorder.

One addition to the recommended guidelines should be that individuals be over the age of 40 years in addition to having 5 years of exposure before yearly screening begins. 


\section{Disclosure}

The author has no known conflicts of interest in this work.

\section{References}

1. Marmor MF, Kellner U, Lai TY, Lyons JS, Mieler WF. Revised recommendations on screening for chloroquine and hydroxychloroquine retinopathy. Ophthmalology. 2011;118(2):415-422.

2. Farrell DF. Unilateral retinitis pigmentosa and cone-rod dystrophy. Clin Ophthalmol. 2009;3:263-270.

3. Grendahl J. Estimation of prognosis and prevalence of retinitis pigmentosa and Usher syndrome in Norway. Clin Genet. 1987;31(4):255-264.

4. Bunker CH, Berson EL, Bromley WC, Hayes RP, Roderick YH. Prevalence of retinitis pigmentosa in Maine. Am J Ophthalmol. 1984;97(3): 357-365.

5. Sen P, Bhargave A, George R, et al. Prevalence of retinitis pigmentosa in South Indian population aged above 40 years. Ophthalmic Epidemiol. 2008;15(4):279-281.

6. Tzekow R. Ocular toxicity due to chloroquine and hydroxychloroquine: electrophysiological and visual function correlates. Doc Ophthalmol. 2005;110(1):111-120

7. Lawrence JS. Prevalence of rheumatoid arthritis. Ann Rheum Dis. 1961; 20:11-17.

8. Symmons D, Turner C, Webb R, et al. The prevalence of rheumatoid arthritis in the United Kingdom: new estimates for a new century. Rheumatology (Oxford). 2002;41(7):793-800.

9. Peschken CA, Esdale JM. Systemic lupus erythematosus in North American Indians: a population based study. J Rheumatol. 2000;27(8): 1884-1891.
10. Helve T. Prevalence and mortality rates of systemic lupus erythematosus and causes of death in SLE patients in Finland. Scand J Rheumatol. 1985;14(1):43-46.

11. Thomas E, Hay EM, Hajeer A, Silman AJ. Sjögren's syndrome: a community-based study of prevalence and impact. BrJ Rheumatol. 1998;37:1069-1076.

12. Nebbioso M, Grenga R, Karavitis P. Early detection of macular changes with multifocal ERG in patients on antimalarial drug therapy. $J$ Ocul Pharmacol Ther. 2009;25(3):249-258.

13. Lai TY, Ngai JW, Chan WM, Lam DS. Visual field and multifocal electroretinography and their correlations in patients on hydroxychloroquine therapy. Doc Ophthalmol. 2006;112(3):177-187.

14. Chang WH, Katz BJ, Warner JE, Vitale AT, Creel D, Digre KB. A novel method for screening the multifocal electroretinogram in patients using hydroxychloroquine. Retina. 2008;28:1478-1486.

15. Chen E, Brown DM, Benz MS, et al. Spectral domain optical coherence tomography as an effective screening test for hydroxychloroquine retinopathy (the "flying saucer" sign). Clin Ophthmalmol. 2010;4: 1151-1158.

16. Kellner U, Renner AR, Tillack H. Fundus autofluorescence and mfERG for early detection of retinal alterations in patients using chloroquine/ hydroxychloroquine. Invest Ophthalmol Vis Sci. 2006;47(8): 3531-3538.

17. Rosenthal AR, Kolb H, Bergsma D, Huxsoll D, Hopkins JL. Chloroquine retinopathy in the rhesus monkey. Invest Ophthalmol Vis Sci. 1978;17(12):1158-1175.
Clinical Ophthalmology

\section{Publish your work in this journal}

Clinical Ophthalmology is an international, peer-reviewed journa covering all subspecialties within ophthalmology. Key topics include: Optometry; Visual science; Pharmacology and drug therapy in eye diseases; Basic Sciences; Primary and Secondary eye care; Patient Safety and Quality of Care Improvements. This journal is indexed on

Submit your manuscript here: http://www.dovepress.com/clinical-ophthalmology-journal

\section{Dovepress}

PubMed Central and CAS, and is the official journal of The Society of Clinical Ophthalmology (SCO). The manuscript management system is completely online and includes a very quick and fair peer-review system, which is all easy to use. Visit http://www.dovepress.com/ testimonials.php to read real quotes from published authors. 